\title{
Produção científica sobre disfagia orofaríngea em idosos nos periódicos brasileiros: uma análise bibliométrica
}

\author{
Scientific production on oropharyngeal \\ dysphagia in elderly in Brazilian journals: \\ a bibliometric analysis
}

\section{Produción científica sobre la disfagia orofaríngea en adultos mayores en periodicos brasileños: analisis bibliométrico}

\author{
Laianna Keyla Virgolino Rodrigues* \\ Leandro Pernambuco*
}

\section{Resumo}

Objetivo: Analisar o perfil da produção científica sobre disfagia orofaríngea em idosos nos periódicos brasileiros, no período de 1995 a 2015. Método: Trata-se de uma pesquisa de revisão bibliográfica, de tipo descritiva, com análise bibliométrica. A busca foi realizada a partir da estratégia de cruzamento dos descritores "idoso", "transtornos de deglutição" e "envelhecimento" e do termo "disfagia", aplicando, em seguida, a seletividade para os critérios de inclusão. Foram considerados quatro grupo de variáveis: identificação da publicação, características dos autores, tipo de estudo e aspectos bibliométricos. Os dados foram submetidos à análise descritiva. Resultado: Foram identificados 9987 artigos, sendo excluídos 9827 por não cumprirem os critérios de inclusão, sendo selecionados 43 artigos para leitura final do texto completo e análise bibliométrica. As instituições com maior número de estudos se encontram na região Sudeste do Brasil, assim como seus autores; a abordagem dos estudos foi majoritariamente quantitativa, seguida da abordagem mista; o tipo de estudo mais encontrado foi o transversal e as amostras concentradas entre 20 e 39 participantes. Os periódicos com maior número de publicações foram os especializados em

\footnotetext{
*Universidade Federal da Paraíba (UFPB), João Pessoa, PB, Brasil.

Contribuição dos autores:

LKVR: coleta de dados, discussão e revisão final do texto;

LP: orientação, discussão e revisão final do texto.
}

E-mail para correspondência: Leandro Pernambuco - leandroape@globo.com Recebido: 25/04/2017

Aprovado: $11 / 09 / 2017$ 
Fonoaudiologia. Conclusão: Neste estudo constatou-se grande polarização das publicações na Região Sudeste, em especial nas universidades públicas do estado de São Paulo. Houve predomínio da abordagem quantitativa e de estudos transversais, com maior produção sobre o tema na área da Fonoaudiologia.

Palavras-chave: Transtornos de deglutição; Idoso; Envelhecimento; Bibliometria.

\section{Abstract}

Objective: To analyze the profile of the scientific production of oropharyngeal dysphagia in the elderly in Brazilian journals, from 1995 to 2015. Method: This is a literature review, descriptive, with bibliometric analysis. The search was carried out from the intersection of strategy descriptors (MeSH): "old," "swallowing disorders" and "aging" and free term "dysphagia", applying then the selectivity to the inclusion criteria. The variables group was: relating to the identification of the publication, characteristics of the authors, study type and bibliometric aspects. The data were submitted to descriptive analysis. Results: 9987 articles were identified, 9827 were excluded for not meeting the inclusion criteria, and 43 were selected for the final reading of the full text and bibliometric analysis. The institutions that had a greater number of studies are in the Southeast region of Brazil, as well as its authors; the approach of the study was mainly quantitative, followed by mixed approach; the type of study most found was the cross and the samples concentrated between 20 and 39 participants. Journals with the most publications were specialized in Speech, Language and Hearing Sciences. Conclusions: In this study, it was found a large polarization of the publications in the Southeast, especially in public universities in the state of São Paulo. There is a predominance of the quantitative approach and cross-sectional studies, with greater scientific production on the subject in the field of Speech, Language and Hearing Sciences.

Keywords: Deglutition disorders; Aged; Aging; Bibliometrics.

\section{Resumen}

Analizar el perfil de la produción científica sobre la disfagia orofaríngea en adultos mayores en la literatura brasileña, de 1995 a 2015. Método: Se trata de una revisión bibliográfica, descriptiva, con análisis bibliométrico. La búsqueda se llevó a cabo a partir de la intersección de los descriptores "adulto mayor", "trastornos de la deglución" y "envejecimiento" y del término "disfagia", aplicando a continuación, la selección para los criterios de inclusión. Cuatro grupo de variables fueron considerados: identificación de la publicación, características de los autores, tipo de estudio y aspectos bibliométricos. Los datos se sometieron a análisis descriptivo. Resultados: Se identificaron 9.987 artículos, 9.827 fueron excluidos por no cumplir con los criterios de inclusión, y se seleccionaron 43 artículos para la lectura final del texto completo y el análisis bibliométrico. Las instituciones con mayor número de estudios se encuentran en la región sudeste de Brasil, así como sus autores; el enfoque de los estudios fue principalmente cuantitativa seguido de enfoque mixto; el tipo de estudio más encontrado fue el transversal y las muestras concentradas entre 20 y 39 participantes. Los periodicos con mayor numero de publicaciones fueron los especializados en Fonoaudiologia. Conclusión: En este estudio se encontró una gran polarización de las publicaciones en la region sudeste, especialmente en las universidades públicas en el estado de Sao Paulo. Hubo un predominio de enfoque cuantitativo y estudios transversales con un mayor producción sobre el tema en el área de la Fonoaudiologia.

Palabras clave: Trastornos de la deglución; Adulto mayor; Envejecimiento; Bibliometría. 


\section{Introdução}

O crescimento da população idosa no Brasil tem despertado o interesse dos profissionais quanto ao cuidar gerontológico. ${ }^{1}$ Neste sentido, a compreensão das modificações fisiológicas resultantes do processo de envelhecimento têm sido foco de estudo na área da saúde. ${ }^{2}$

No envelhecimento ocorrem mudanças naturais no funcionamento da dinâmica da deglutição, e surgem com mais frequência doenças específicas ou quadros degenerativos que podem interferir nessa função, a exemplo do acidente vascular encefálico, traumatismo crânioencefálico, câncer de cabeça e pescoço, tumores cerebrais e demências. ${ }^{3-4}$ Todo esse cenário pode tornar a deglutição do idoso menos eficiente, contribuindo para o aparecimento da disfagia orofaríngea. ${ }^{5}$

As características da deglutição em idosos são diversas e incluem dificuldades de controle e ingestão do bolo, diminuição da força mastigatória, aumento da quantidade de tecido conjuntivo na língua, acúmulo de resíduos, paresia dos músculos faríngeos, redução do grau de elevação da laringe, atraso no início da excursão hiolaríngea, necessidade de deglutições múltiplas, maior ocorrência de tosse, movimentos laríngeos polifásicos, maior probabilidade de aspiração após a deglutição e aumento de duração da fase esofágica. ${ }^{6}$ Além dos aspectos anatomofisiológicos, também devem ser consideradas as alterações no prazer ao alimentar-se e na socialização. ${ }^{?}$

No Brasil, o envolvimento de pesquisadores com a disfagia orofaríngea passou a ocorrer com maior ênfase a partir da década de 90, mesmo período em que as discussões sobre as especialidades na área da Fonoaudiologia foram enfatizadas, culminando com a publicação da primeira Resolução sobre o tema em 1996, a qual estabeleceu a Motricidade Oral como área de especialidade da Fonoaudiologia ${ }^{8}$ e que, mais adiante, daria desdobramento à Disfagia como especialidade, conforme resolução do Conselho Federal de Fonoaudiologia no ano de $2010 .{ }^{9}$

Apesar de a produção científica ser robusta e ter tomado maior proporção recentemente, ainda é necessário muito investimento, estudo e dedicação ao tema. ${ }^{10}$ Nesse contexto, a bibliometria se revela como opção a ser utilizada como metodologia para a obtenção de indicadores de avaliação da produção científica, uma vez que analisa a produtividade científica por meio do estudo quantitativo e objetiva desenvolver indicadores que possam traduzir um panorama da atualidade. ${ }^{11-12}$

Dessa forma, este estudo tem como principal objetivo analisar o perfil da produção científica sobre disfagia em idosos nos periódicos brasileiros, no período entre janeiro de 1995 e dezembro de 2015.

\section{Método}

Trata-se de uma pesquisa de revisão bibliográfica, de tipo descritiva, com análise bibliométrica da produção científica dos periódicos nacionais entre janeiro de 1995 e dezembro de 2015, indexados nas bases de dados Medline, LILACS e SciELO. Adotaram-se como critérios de inclusão os artigos originais, de revisão, estudos de caso, séries de casos, ensaios, artigos de opinião, de atualização, de reflexão e de comunicação breve, publicados nos periódicos nacionais durante o período descrito. Excluíram-se os anais de congresso, resenhas, editoriais, cartas ao editor, dissertações e teses.

A busca foi realizada em cada base de dados separadamente, no idioma português, a partir da estratégia de cruzamento de descritores oficiais (DeCS), e do termo livre "disfagia", sendo este utilizado devido à relevância ao tema abordado neste estudo. Os cruzamentos foram os seguintes: "envelhecimento x transtornos de deglutição"; "envelhecimento x disfagia"; "idoso x transtornos de deglutição" e "idoso x disfagia", aplicando-se em seguida, a seletividade de acordo com os critérios de inclusão.

A coleta nas bases de dados foi realizada por dois pesquisadores e os casos que geraram dúvida foram discutidos com um terceiro pesquisador para decisão consensual. Neste estudo foram considerados quatro grupo de variáveis, de acordo com estudo anterior ${ }^{13}$ : identificação da publicação, autoria, tipo de estudo e características bibliométricas. Primeiramente, foi procedida leitura do título e resumo, e, na etapa seguinte, a leitura do texto completo até alcançar o número final de artigos incluídos.

Em relação às informações de identificação, foram considerados ano de publicação, região e estado nos quais os estudos foram realizados. As variáveis referentes à autoria foram número de instituições envolvidas, número de autores por ar- 
tigo e financiamento. Além disso, foram coletadas informações sobre a formação do primeiro autor.

Para conhecer a formação acadêmica, foram pesquisados os currículos dos autores na Plataforma Lattes, e em caso de possuir mais de um curso de graduação, considerou-se aquele mais vinculado à temática do artigo. Informações específicas sobre o trabalho foram obtidas mediante leitura do resumo e texto completo. Foram também coletadas informações referentes à abordagem (quantitativa, qualitativa e mista), desenho de estudo (estudo de caso, transversal, coorte, ensaio clínico, ecológico, caso-controle, revisão, tradução/validação de questionários), número da amostra total de cada estudo (para os estudos de revisão, a amostra foi considerada como o número de artigos analisados no estudo), e os periódicos nos quais os estudos foram publicados. Os dados foram analisados em dois períodos de tempo (1995-2005 e 2006-2015) de acordo com sua distribuição absoluta e relativa.

\section{Resultados}

No período 1995 a 2015, foram identificados 9987 artigos. Destes, 9827 foram excluídos por não cumprirem os critérios de elegibilidade. Ao todo, foram selecionados 43 artigos para leitura final do texto completo e análise bibliométrica, de acordo com o fluxograma apresentado na Figura 1.

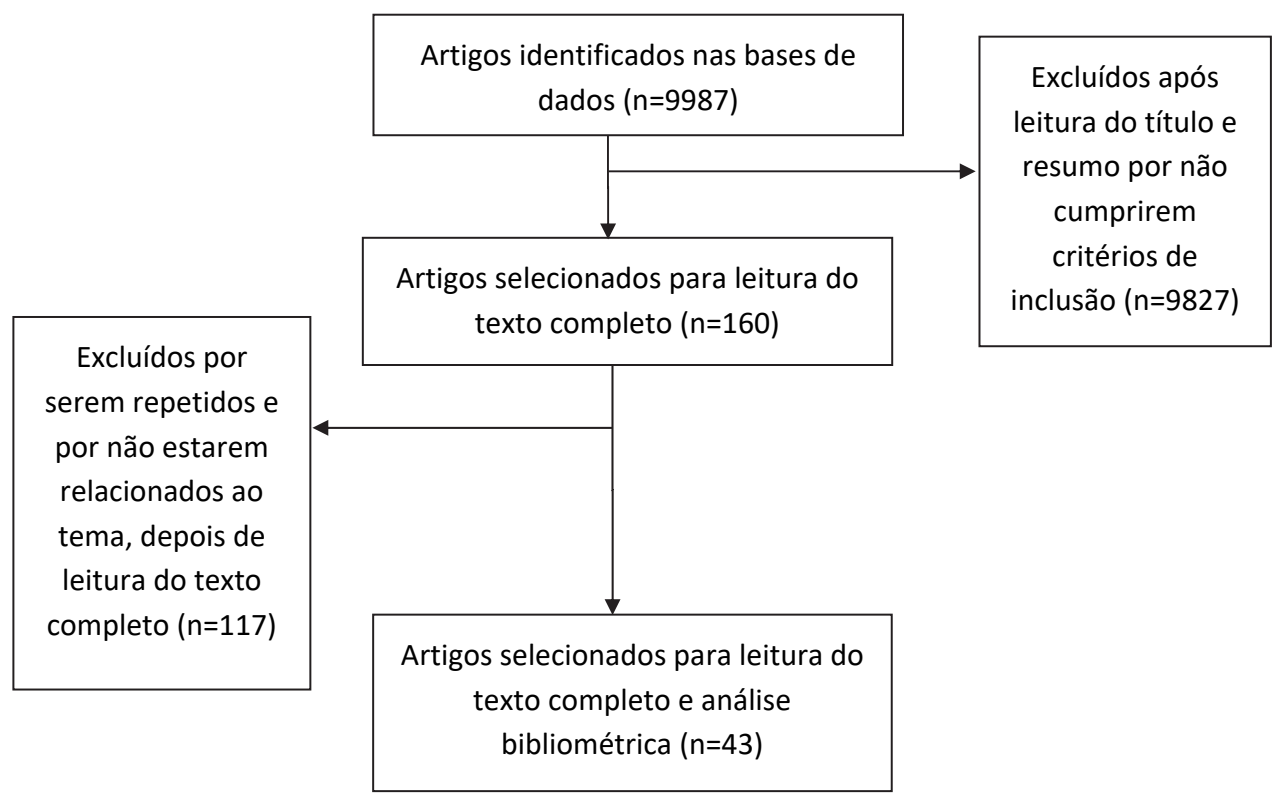

Figura 1. Fluxograma para seleção dos estudos sobre disfagia no idoso publicados entre 1995 e 2015.

Na Tabela 1 observam-se os resultados das variáveis referentes à autoria dos artigos, com exceção das variáveis região e estado que, neste caso, referem-se ao local de realização do estudo. A região Sudeste se destaca com o maior número de manuscritos, ao contrário da região Norte que não apresentou nenhuma publicação sobre o tema no período estudado. O estado de São Paulo lidera com larga vantagem em número de publicações, com sua produção quase duplicada entre o primeiro e o segundo período considerado nesta revisão.

Os autores que mais publicaram estavam vinculados à Universidade Federal de São Paulo (UNIFESP) e Universidade de São Paulo (USP), com destaque para estudos realizados por três ou quatro autores, sendo estes, em sua maioria, fonoaudiólogos. A análise das instituições que produziram os manuscritos foi feita de acordo com 
Tabela 1. Análise descritiva das variáveis referentes à autoria dos estudos sobre disfagia no idoso publicados em periódicos brasileiros no período entre 1995 e 2015.

\begin{tabular}{|c|c|c|c|c|c|c|}
\hline \multirow{2}{*}{ Variáveis } & \multicolumn{2}{|c|}{ 1995-2005 } & \multicolumn{2}{|c|}{$2006-2015$} & \multicolumn{2}{|c|}{ TOTAL } \\
\hline & $\mathbf{n}$ & $\%$ & $\mathbf{n}$ & $\%$ & $\mathbf{n}$ & $\%$ \\
\hline \multicolumn{7}{|l|}{ Região } \\
\hline Norte & 0 & 0 & 0 & 0 & 0 & 0 \\
\hline Nordeste & 0 & 0 & 2 & 6 & 2 & 5 \\
\hline Centro-oeste & 0 & 0 & 1 & 3 & 1 & 2 \\
\hline Sudeste & 9 & 90 & 21 & 64 & 30 & 70 \\
\hline Sul & 1 & 10 & 6 & 18 & 7 & 16 \\
\hline Não informado & 0 & 0 & 3 & 9 & 3 & 7 \\
\hline \multicolumn{7}{|l|}{ Estado } \\
\hline São Paulo & 9 & 90 & 16 & 49 & 25 & 59 \\
\hline Rio Grande do Sul & 1 & 10 & 3 & 9 & 4 & 9 \\
\hline Rio de Janeiro & 0 & 0 & 4 & 12 & 4 & 9 \\
\hline Minas Gerais & 0 & 0 & 1 & 3 & 1 & 2 \\
\hline Paraná & 0 & 0 & 3 & 9 & 3 & 7 \\
\hline Distrito Federal & 0 & 0 & 1 & 3 & 1 & 2 \\
\hline Alagoas & 0 & 0 & 2 & 6 & 2 & 5 \\
\hline Não informado & 0 & 0 & 3 & 9 & 3 & 7 \\
\hline \multicolumn{7}{|l|}{ Instituições } \\
\hline USP & 2 & 13 & 8 & 18 & 10 & 17 \\
\hline UFRJ & 0 & 0 & 2 & 4 & 2 & 3 \\
\hline PUC-SP & 4 & 27 & 4 & 9 & 8 & 14 \\
\hline UNCISAL-AL & 0 & 0 & 2 & 4 & 2 & 3 \\
\hline UNIFESP & 5 & 33 & 5 & 11 & 10 & 17 \\
\hline UNESP & 1 & 7 & 5 & 11 & 6 & 10 \\
\hline Outras & 3 & 20 & 19 & 43 & 22 & 36 \\
\hline \multicolumn{7}{|c|}{ Número de instituições } \\
\hline 1 & 7 & 64 & 20 & 63 & 27 & 62 \\
\hline 2 ou mais & 4 & 36 & 12 & 37 & 16 & 38 \\
\hline \multicolumn{7}{|l|}{ Número de autores } \\
\hline $1-2$ & 0 & 0 & 8 & 24 & 8 & 19 \\
\hline $3-4$ & 7 & 70 & 15 & 45 & 22 & 51 \\
\hline 5 ou mais & 3 & 30 & 10 & 31 & 13 & 30 \\
\hline \multicolumn{7}{|l|}{ Financiamento } \\
\hline Sim & 1 & 10 & 1 & 3 & 2 & 4 \\
\hline Não & 9 & 90 & 32 & 97 & 41 & 96 \\
\hline \multicolumn{7}{|c|}{ Formação do $1^{\circ}$ autor } \\
\hline Fonoaudiólogo & 8 & 80 & 23 & 70 & 31 & 72 \\
\hline Nutricionista & 0 & 0 & 4 & 12 & 4 & 9 \\
\hline Médico & 2 & 20 & 5 & 15 & 7 & 17 \\
\hline Odontólogo & 0 & 0 & 1 & 3 & 1 & 2 \\
\hline
\end{tabular}

a vinculação de cada autor e em virtude dos estudos interinstitucionais, o número total dessa variável ficou acima do total de publicações encontradas.

A Tabela 2 mostra a abordagem, desenho, e amostra do trabalho. A abordagem foi majoritariamente quantitativa, seguida da abordagem mista; ambas com aumento progressivo no número de publicações entre os dois períodos. Os estudos transversais foram mais frequentes e triplicaram em número nesse período. A maioria dos estudos apresentou amostra concentrada entre 20 e 39 participantes, com crescimento progressivo desse número ao longo do período analisado pelo estudo. 
Tabela 2. Análise descritiva das variáveis referentes a aspectos metodológicos dos estudos sobre disfagia no idoso publicados em periódicos brasileiros no período entre 1995 e 2015.

\begin{tabular}{|c|c|c|c|c|c|c|}
\hline & \multicolumn{2}{|c|}{ 1995-2005 } & \multicolumn{2}{|c|}{ 2006-2015 } & \multicolumn{2}{|c|}{ TOTAL } \\
\hline & $\mathbf{n}$ & $\%$ & $\mathbf{n}$ & $\%$ & $\mathbf{n}$ & $\%$ \\
\hline \multicolumn{7}{|l|}{ Abordagem } \\
\hline Quantitativa & 3 & 30 & 18 & 55 & 21 & 49 \\
\hline Qualitativa & 4 & 40 & 5 & 15 & 9 & 21 \\
\hline Mista & 3 & 30 & 10 & 30 & 13 & 30 \\
\hline \multicolumn{7}{|l|}{ Desenho do estudo } \\
\hline Transversal/Série de casos (observacional) & 8 & 80 & 25 & 70 & 31 & 73 \\
\hline Revisão & 0 & 0 & 1 & 3 & 1 & 2 \\
\hline Série de casos (intervenção) & 1 & 10 & 3 & 9 & 4 & 9 \\
\hline Estudo de Caso & 1 & 10 & 3 & 9 & 4 & 9 \\
\hline Grupo-controle & 0 & 0 & 3 & 9 & 3 & 7 \\
\hline \multicolumn{7}{|l|}{ Amostra } \\
\hline 1 a 19 & 4 & 40 & 8 & 25 & 12 & 28 \\
\hline 20 a 39 & 2 & 20 & 12 & 36 & 14 & 33 \\
\hline 40 a 59 & 0 & 0 & 6 & 18 & 6 & 14 \\
\hline 60 a 79 & 0 & 0 & 3 & 9 & 3 & 7 \\
\hline 80 a 99 & 0 & 0 & 0 & 0 & 0 & 0 \\
\hline 100 ou mais & 4 & 40 & 4 & 12 & 8 & 18 \\
\hline
\end{tabular}

No gráfico 1 constam as revistas com maior número de manuscritos em relação ao tema. Nesse âmbito, a revista "Distúrbios da Comunicação" lidera e mantem sua média de publicações entre a primeira e a segunda década do estudo. Em segundo lugar em número de publicações está o Jornal da Sociedade Brasileira de Fonoaudiologia/CoDAS, seguido pela Revista Brasileira de Geriatria e Gerontologia, Arquivos de Gastroenterologia e Arquivos de Neuropsiquiatria, com o mesmo número de publicações sobre o tema. As revistas que apresentaram apenas uma publicação durante o período do estudo foram classificadas na categoria "outras".

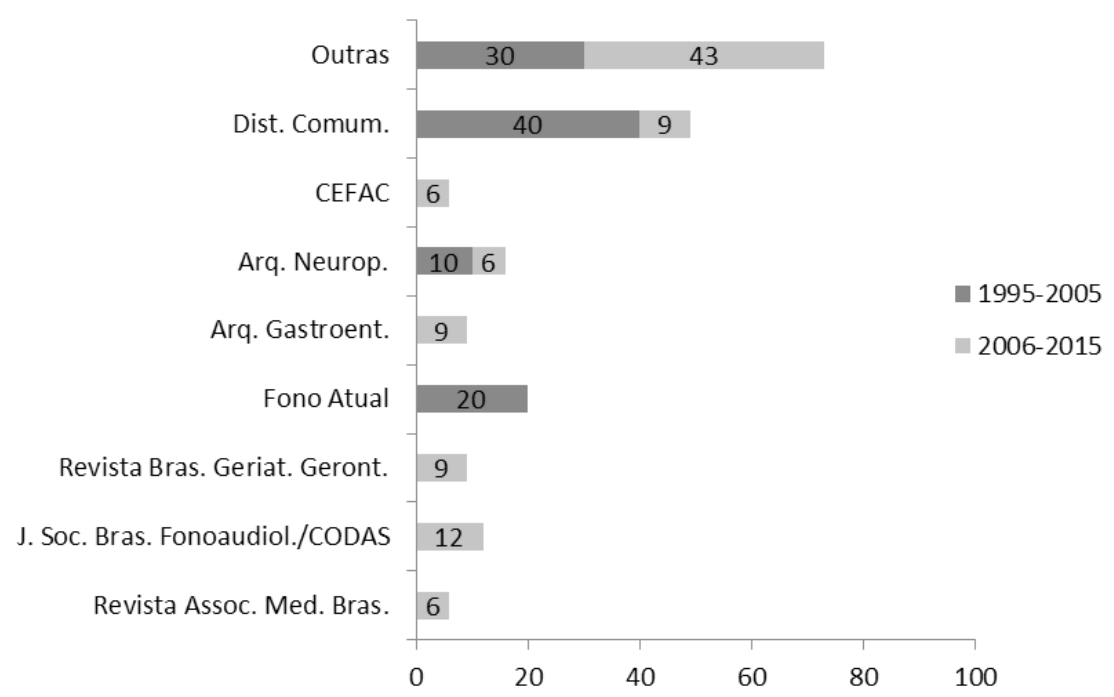

Figura 2. Distribuição percentual das publicações sobre disfagia no idoso de acordo com o periódico nacional, no período entre 1995 e 2015. 


\section{Discussão}

Neste estudo, constatou-se que o maior número de publicações ficou concentrado na região Sudeste e no estado de São Paulo. Além disso, a quantidade de publicações nesses locais aproximadamente dobrou entre as duas décadas investigadas, ao contrário da região Norte que não apresentou nenhuma publicação nesse período.

Identifica-se uma estreita relação entre a publicação desses manuscritos com o ensino superior e, principalmente, com os programas de pós-graduação, fato este que corrobora o desenvolvimento das universidades de forma localizada e centralizada em nosso país, especialmente na Região Sudeste. ${ }^{14}$ Esse panorama é justificado pelo fato de que o ensino superior no Brasil iniciou sua organização mais sistemática a partir de 1934, com a fundação da Universidade de São Paulo. Esse cenário reflete uma condição histórica recente no âmbito da Educação, bem como o desenvolvimento de outras instituições de forma centralizada nessa região. ${ }^{14-15}$

A concentração de estudos realizados na região Sudeste e em especial no estado de São Paulo parece ser resultado de um polo expressivo de universidades e faculdades instaladas nessa região. De acordo com o $\mathrm{MEC}^{16}$, a região Sudeste abarca $49 \%$ de todas IES (Instituição de Ensino Superior) brasileiras. Por outro lado, a região Norte detém a menor concentração de instituições, representando apenas $6 \%$ da totalidade do País. A região Nordeste possui 432 IES, representando $18 \%$ da totalidade, assumindo assim o segundo lugar em número de IES, especialmente em razão de fenômeno recente de investimento no ensino superior nessa região ${ }^{17}$; ainda no Nordeste, as IES estão concentradas nas capitais dos estados, especialmente em Sergipe e Piauí, com $86 \%$ e $70 \%$ do total de suas IES localizadas nas capitais, respectivamente. A região Nordeste é seguida pelas Regiões Sul e Centro-Oeste, consecutivamente.

Quanto às instituições que mais produziram, também merece destaque as IES do estado de São Paulo, visto que mais uma vez a concentração das publicações se encontra nas universidades desse estado.

Observou-se que a maioria dos autores estavam vinculados à USP e UNIFESP, ambas com o mesmo número de publicações. Esta última mantem os cursos ministrados na área da saúde ${ }^{18} \mathrm{e}$ desde 2005 conta com o apoio das prefeituras locais e os recursos provenientes do programa de expansão do governo federal. Além disso, ampliou seu número de professores com titulação mínima de doutorado e passou a oferecer novos cursos de graduação, que se fundamentam em modernos projetos pedagógicos e permitem maior flexibilidade curricular; acarretando, portanto, maior estímulo a novas pesquisas. ${ }^{18}$

Fato importante é que a UNIFESP manteve número semelhante de publicações sobre o tema entre o primeiro e segundo períodos estudados, ao passo que a USP quadruplicou seu número de publicações entre os períodos.

De acordo com o ranking da THE (Times Higher Education) $)^{19}$, divulgado em 2015, entre as 800 universidades com melhor classificação, 17 são brasileiras. A lista abarca instituições de 70 países e representa $4 \%$ da elite acadêmica global. A USP foi enquadrada na faixa classificatória 201-250, liderando o ensino superior brasileiro e latino-americano. A UNIFESP, no entanto, não esteve presente nesse ranking.

Ainda sob esta ótica, em relação ao número de instituições envolvidas, de acordo com a autoria, é importante constatar que a maioria dos estudos envolveu apenas uma instituição, seguida dos estudos envolvendo duas ou mais instituições distintas. Destaca-se que em ambos os casos os manuscritos praticamente triplicaram entre a primeira e a segunda década do estudo.

Esse perfil sugere evolução em direção a um modelo de produção de conhecimento coletivo e colaborativo, na medida em que os diversos produtores participam de arranjos, redes e alianças entre as academias, as empresas e o setor público. ${ }^{20}$ Esse modelo implica num novo acordo entre academia e sociedade, no qual diversos atores sociais como empresas, entidades públicas e ONGs passam a exercer papel mais ativo e direto na produção do conhecimento. ${ }^{21}$

No que se refere ao número de autores por estudo, constatou-se maioria de artigos publicados com três ou quatro autores, seguidos dos manuscritos com cinco ou mais autores e pequena quantidade com autoria de um ou dois autores. Tal cenário se justifica devido a uma crescente tendência de integração entre os saberes de distintos autores e formações. Autores relatam que a capacidade de criação de conhecimento dos autores está diretamente relacionada com a sua interação com outros 
atores, num processo de aprendizagem e trocas coletivas de conhecimento. ${ }^{20}$

A aprendizagem através da cooperação e da construção da confiança mútua cresce também com a evolução dos relacionamentos. Esses elementos constituem um recurso compartilhado que tende a superar eventuais antagonismos com a elaboração de projetos e programas integrados. ${ }^{20}$

Embora a maioria das publicações tenha sido com três ou quatro autores, é importante ficar atento ao fato de que o número de publicações com apenas um ou dois autores foi o que obteve o maior crescimento entre as décadas, seguidos dos estudos com cinco ou mais autores.

No que concerne à metodologia dos trabalhos analisados, a maioria deles apresentou abordagem quantitativa, do tipo transversal e com amostras pequenas de 20 a 39 sujeitos. A pesquisa quantitativa permite a mensuração de opiniões, reações, hábitos e atitudes de um determinado universo ${ }^{22}$ e vem sendo amplamente utilizada nas pesquisas com a população idosa, visando sua maior praticidade e agilidade, não demandando de grande espaço de tempo, periodicidade e assiduidade dos participantes ao método.

Idosos, em geral, não possuem boa adesão aos tratamentos e estudos que exijam acompanhamentos periódicos e por longo período de tempo, devido a fatores como dificuldade de acesso e locomoção, tempo prolongado para atendimento e consultas, problemas sociais, presença de enfermidades e falta de inclusão dos familiares na orientação e acompanhamento; o que por sua vez, diminuem as chances de adesão ao método qualitativo. Este fato se torna ainda mais frequente em idosos com distúrbios de deglutição que, em sua grande maioria, não reconhecem a sua dificuldade ou incapacidade em deglutir, e não procuram o tratamento especializado.

No entanto, é válido ressaltar que o método qualitativo é útil e necessário para identificar e explorar os significados dos fenômenos estudados e as interações que estabelecem, possibilitando assim, estimular o desenvolvimento de novas compreensões sobre a variedade e a profundidade dos fenômenos sociais. ${ }^{23}$. As abordagens podem ser complementares, visando à minimização da subjetividade e a aproximação do objeto de estudo e pesquisadores, e proporcionando maior confiabilidade aos dados. ${ }^{24}$
Outra questão diz respeito às condições de pesquisas por ensaios clínicos randomizados, nos quais muitas vezes ocorre controle excessivo sobre a amostra, não retratando condições de rotina, o que dificulta bastante a adesão do público idoso. Desta forma, os achados dos ensaios clínicos randomizados podem ser complementados por estudos transversais. ${ }^{25}$

O estudo transversal representa um corte instantâneo da população por meio de uma amostragem, examinando-se nos integrantes da casuística ou amostra, a presença ou ausência da exposição e a presença ou ausência do efeito (ou doença). Possui como principais vantagens o fato de ser de baixo custo, e por praticamente não haver perdas de seguimento ${ }^{26}$, fato que justificaria a imensa maioria de estudos realizados nessa proposta, inclusive com frequência triplicada entre as duas décadas consideradas nesta revisão. Além disso, nesse tipo de estudo não há existência de um período de seguimento, o que pode representar uma opção mais segura para os pesquisadores em termos de perdas, considerando que a população idosa está mais próxima da finitude e com maior susceptibilidade a óbitos. ${ }^{27}$

Outro aspecto diz respeito ao apoio financeiro para os estudos. No que concerne aos artigos publicados com apoio financeiro, os números são lamentavelmente baixos, representados, neste estudo, por uma parcela ínfima das publicações nas duas décadas do estudo. Esse panorama demostra o ainda insuficiente incentivo na publicação de novas pesquisas, justificado, em parte, devido ao financiamento público ter iniciado seu desenvolvimento significativo somente a partir da década de 1990 , tornando o fomento à pesquisa insuficiente. ${ }^{28}$

No Brasil, um dos maiores obstáculos para o desenvolvimento das pesquisas é o custo elevado de projetos de qualidade para sua execução, assim como a demanda de tempo e dedicação por parte dos pesquisadores, que no geral não recebem bolsas compatíveis para se dedicarem exclusivamente aos projetos. ${ }^{29}$

Esses estudos se tornam ainda mais onerosos quando realizados com a população idosa, visto sua vulnerabilidade e limitações funcionais e físicas, e, principalmente nos idosos com distúrbios de deglutição, pois estes demandam de maiores recursos de avaliação e terapêutica a serem utilizados. A avaliação clínica, por sua vez, compreende questões relacionadas a hábitos alimentares, uso de medica- 
mentos, aspectos dentários, musculatura orofacial, estruturas estomatognáticas e avaliação funcional da deglutição em diversas consistências. Além disso, utiliza-se de exames como a videofluroscopia, com o objetivo de comparar seus resultados aos da avaliação clínica ou, ainda, a videoendoscopia da deglutição, numa análise comparada, ou somente esse exame como avaliação instrumental. ${ }^{30}$ A falta de recursos para cobrir esses custos se mantem presente durante a terapêutica e interfere na realização desse tipo de estudo nessa população. Custos esses que se mantem presentes durante o processo de reabilitação e prejudicam cada vez mais a realização destes.

Em relação à formação do primeiro autor, a Fonoaudiologia lidera com ampla vantagem as autorias das publicações, o que se justifica devido ser a "Disfagia" e a "Gerontologia" áreas de especialidade reconhecidas pelo conselho de classe da Fonoaudiologia, despertando de maneira crescente maior interesse sobre esses temas. Além disso, é importante destacar que outras áreas de atuação vêm em ascendência no número de publicações sobre o tema, a exemplo de Nutrição, Odontologia e Medicina, especialmente a Gastroenterologia e Otorrinolaringologia; visto que são áreas de interesses afins com a Fonoaudiologia em diversos aspectos.

No que se refere ao número de publicações sobre o tema por Revistas, a "Distúrbios de Comunicação" apresentou maior número de estudos publicados, apesar de manter seu número de manuscritos praticamente iguais entre as duas décadas do estudo. Esta revista é uma publicação científica do Curso de Fonoaudiologia, Pós Graduação em Fonoaudiologia e DERDIC (Divisão de Educação e Reabilitação dos Distúrbios da Comunicação) da PUC-SP.

Dentre as revistas que mais publicaram sobre o tema, as duas com maior número de manuscritos são de Fonoaudiologia, outra é um periódico especializado que publica no âmbito da Geriatria e Gerontologia e outras têm enfoque médico, porém com associação a temas inerentes a Fonoaudiologia.

É oportuno destacar que o crescimento da produção científica precisa de maior engajamento das mais diversas áreas para produção de conhecimento. As produções necessitam de maior fomento e incentivo, visto que o número de publicações sobre o tema em periódicos nacionais ainda é demasiadamente baixo quando comparado aos periódicos internacionais. Devido à falta de estímulo e visibilidade dos periódicos nacionais, os autores preferem, em sua maioria, publicarem seus estudos em periódicos internacionais de maior impacto e reconhecimento, o que reforça progressivamente maior descrédito dos periódicos nacionais.

\section{Conclusão}

Neste estudo, constatou-se uma grande polarização das publicações sobre disfagia em idosos na Região Sudeste, em especial, nas Universidades públicas do Estado de São Paulo.

A abordagem dos estudos foi majoritariamente quantitativa, seguida da abordagem mista e o tipo de estudo predominante foi o transversal. A produção científica sobre o tema revela maior produção de manuscritos na área da Fonoaudiologia, visto que a "Disfagia" e "Gerontologia" são áreas de especialidade dessa categoria, reconhecidas pelos Conselhos de classe.

\section{Referências}

1 Freitas MC, Maruyama SAT, Ferreira TF, Motta AMA. Perspectivas das pesquisas em gerontologia e geriatria: revisão da literatura. Rev Lat Am Enfermagem. 2002; 10(2): 221-8.

2 Yoshida FS, Mituuti CT, Totta T, Berretin FG. A influência da função mastigatória na deglutição orofaríngea em idosos saudáveis. Audiology Communication Research. 2015; 20(2): 161-6.

3 Nasi A. Disfagia no indivíduo idoso. In: Macedo Filho E, Pissan JC, Carneiro J, Gomes G. Disfagia: abordagem multidisciplinar. São Paulo: Frôntis; 1998. p. 47-62.

4 Ekberg O, Feinberg MJ. Videofluoroscopy in elderly patients with aspiration: importance of evaluating both oral and pharyngeal stages of deglutition. AJR Am J Roentgenol. 1991; 156(2): 293-6.

5 Feijó, AV; Rieder, CRM. Distúrbios da Deglutição em Idosos. In: Jacobi JS, Levy DS, Silva LMC. Disfagia: avaliação e tratamento. Rio de Janeiro: Revinter, 2004. p. 225-31.

6 Nilsson H, Ekberg O, Olsson R, Hindfelt B. Quantitative aspects of swallowing in an elderly nondysphagic population. Dysphagia. 1996; 11(3): 180-4.

7 Furkim AM. Disfagia orofaríngea neurogênica. In: Marchesan IC. Fundamentos em fonoaudiologia: aspectos clínicos da motricidade oral. Rio de Janeiro: Guanabara Koogan, 2005. p. $121-32$

8 Ferigotti ACM, Nagib L. Editorial. Fonoaudiologia: reabertas as discussões sobre especialidades. Revista da Sociedade Brasileira de Fonoaudiologia. 2009; 14(3): 8-10. 
9 CFFa: Conselho Federal de Fonoaudiologia. Resolução $\mathrm{CFFa}, \mathrm{N}^{\circ}$ 383, de 20 de março de 2010. [Acesso em 2016 Mai 16]. Disponível em: http://Www.Fonoaudiologia.Org.Br/ Legislacaopdf/Res\%20383-10\%20-\%20disfagia.Pdf

10 Santoro PP. Editorial II - Disfagia orofaríngea: panorama atual, epidemiologia, opções terapêuticas e perspectivas futuras. Revista CEFAC. 2008; 10(2).

11 Silva RC. Avaliação da informação científica em Bibliometria aplicada às Ciências da Saúde. Bibliotecas, informação, usuários: abordagens de transformação para Biblioteconomia e Ciência da Informação: Anais $25^{\circ}$ do Congresso Brasileiro de Biblioteconomia, Documentação e Ciência da Informação FEBAB; 2013 Set 07-10; Santa Catarina, BR; 2013.

12 Mugnaini R. Indicadores bibliométricos da produção científica brasileira: uma análise a partir da base Pascal. Ciência da Informação. 2004; 33(2): 123-31.

13 Roig JJ, Guedes MBOG, Silva JMD, Lima KC. Analysis of the scientific production of the Brazilian Journal of Geriatrics and Gerontology: a bibliometric review. Revista Brasileira de Geriatria e Gerontologia. 2014; 17(3): 659-71.

14 Franco AP. Ensino Superior no Brasil: cenário, avanços e contradições. Jornal de políticas educacionais. 2008; 2(4): 53-63.

15 Cunha LA. Desenvolvimento desigual e combinado no ensino superior: estado e mercado. Educação \& Sociedade. 2004; 25(88): 795-817.

16 Alvarez AMT. Projeto CNE/UNESCO 914BRZ1136.3 "Desenvolvimento, aprimoramento e consolidação de uma educação nacional de qualidade". São Paulo: 2013 [Acesso 2015 nov 21]. Disponível em http://portal.mec.gov.br/index. php?option $=$ com_docman $\&$ view $=$ download $\&$ alias $=13944$ produto-1-senso-educ-superior-pdf\&Itemid $=30192$

17 Ministério Da Educação. Instituto Nacional de Estudos e Pesquisas Educacionais Anísio Teixeira. Resumo TécnicoCenso Da Educação Superior 2008 (Dados Preliminares). Distrito Federal: 2009. [Acesso 2016 Mai 17]. Disponível em: http://download.inep.gov.br/download/censo/2008/resumo tecnico_2008_15_12_09.pdf

18 Brasil. Universidade Federal de São Paulo. Apresentação. São Paulo: 2014. [Acesso 2015 Dez 17]. Disponível em: http:// www.unifesp.br/institucional/institucionalsub/apresentacao
19 THE (Times Higher Education) World University Rankings. World University Rankings 2015-2016. 2016. [Acesso 2016 Jan 12]. Disponível em: https://www.timeshighereducation. com/world-university-rankings/2016/worldranking\#!/page/20/ length/25/country/116/sort_by/rank_label/sort_order/asc/cols/ rank_only

20 Gazda E, Quandt CO. Colaboração interinstitucional em pesquisa no Brasil: tendências em artigos na área de gestão da inovação. RAE eletrônica. 2010; 9(2).

21 Martin BR. The changing social contract for science and the evolution of the university. In: Geuna A, Salter AJ, Steinmueller WE. Eds. Science and Innovation: Rethinking the Rationales for Funding and Governance. Cheltenham: Edward Elgar Publishing, 2003. p. 7-29.

22 Terence ACF, Filho EE. Abordagem quantitativa, qualitativa e a utilização da pesquisa-ação nos estudos organizacionais; 2006 Out 09-11; Fortaleza, BR; 2006.

23 Bartunek JM, Seo M. Qualitative research can add new meanings to quantitative research. Journal of Organizational Behavior. 2002; 23(2): 237-42

24 Godoy AS. Introdução à pesquisa qualitativa e suas possibilidades. Revista de Administração de Empresas.1995; 35(2): 57-63.

25 Santos IS, Victora CG. Serviços de saúde: epidemiologia, pesquisa e avaliação. Cad Saúde Pública. 2004;20(2):337-41.

26 Haddad N. Metodologia de estudos em ciências da saúde. 1ed. São Paulo: Roca, 2004.

27 Fonseca, AMG. Uma abordagem psicológica da passagem à reforma - desenvolvimento, envelhecimento, transição e adaptação. [Tese]. Porto: Universidade do Porto; 2004.

28 Corbucci PR. Desafios da educação superior e desenvolvimento no Brasil. Texto para Discussão. 2007; 1287: $1-33$

29 Steenhagen CHVA, Motta LB. Deglutição e envelhecimento: enfoque nas manobras facilitadoras e posturais utilizadas na reabilitação do paciente disfágico. Revista Brasileira de Geriatria e Gerontologia. 2006; 9(3): 89-100.

30 Acosta NB, Cardoso MCAF. Presbifagia: estado da arte da deglutição do idoso. Revista de Brasileira de Ciências do Envelhecimento Humano. 2012; 9(1): 143-54. 